\title{
Effect of SLC34A2 gene mutation on extracellular phosphorus transport in PAM alveolar epithelial cells
}

\author{
TIANGANG MA, DANHUA QU, BINGDI YAN, QINGHUA ZHANG，JIN REN and YANBING HU \\ Department of Pneumology, The Second Hospital of Jilin University, Changchun, Jilin 130041, P.R. China
}

Received June 28, 2017; Accepted October 17, 2017

DOI: $10.3892 /$ etm.2017.5380

\begin{abstract}
A mutation in the IIb sodium phosphate transporter SLC34A2 gene has recently been described in pulmonary alveolar microlithiasis (PAM) patients. Experiments in this study were aimed at confirming the role of the gene product in PAM by comparing phosphorylated products in extracellular fluid of alveolar epithelial cells overexpressing the SLC34A2 gene or its mutated version. Eukaryotic expression vectors were constructed and transfected into A549 human alveolar epithelial cells. There were three groups of cells including those transfected with empty vector plasmid pcDNA3.1(+) (plasmid control group), those transfected with normal SLC34A2 gene expressed from pcDNA3.1 (normal control group), and those transfected with a version of the PAM SLC34A2 gene linked to the pcDNA3.1(+) (PAM group). Transfection efficiencies were detected by reverse transcription-polymerase chain reaction (RT-PCR). At $48 \mathrm{~h}$ after transfection, the concentration of inorganic phosphorus in the culture medium was detected using an automatic biochemical analyzer. Our results showed the concentration of inorganic phosphorus in the supernatant of the normal control group was significantly lower than that in the plasmid control and PAM groups $(\mathrm{P}<0.01)$, and the concentration in the PAM group was significantly lower than that in the plasmid control group $(\mathrm{P}<0.01)$. Based on our findings it is possible that the SLC34A2 gene mutation is the cause of the pathogenic changes observed in PAM patients, given that the function of the phosphate transporter seems to be affected and it is conceivable that it would lead to extracellular fluid alterations in vivo.
\end{abstract}

\section{Introduction}

Pulmonary alveolar microlithiasis (PAM) is a long-term disease, which progresses slowly, producing a gradual decline

Correspondence to: Dr Yanbing Hu, Department of Pneumology, The Second Hospital of Jilin University, 218 Ziqiang Street, Nanguan, Changchun, Jilin 130041, P.R. China

E-mail: huyanbing1985@163.com

Key words: SLC34A2 gene mutation, pulmonary alveolar microlithiasis, eukaryotic expression vector, phosphate in lung function. The disease can show a familial distribution in approximately $38-61 \%$ of cases, belonging to the group of autosomal recessive genetic diseases (1). X-ray energy dispersive spectroscopy of the lung suggests that the ratio of phosphorus to calcium ions in the microlithiasis observed in patients with PAM is $1: 2$, which is equivalent to the ratio in calcium phosphate and hydroxyapatite, suggesting that the main component in the microlithiasis is phosphate. The SLC34A2 gene encodes a type IIb sodium phosphate co-transporter (NaPi-IIb), involved in the metabolism of inorganic phosphorus in vivo (2).

A c575C $>$ A homozygous mutation (T192K phenotype) has been found in all of three patients of a family affected by PAM, by sequencing all the exon PCR products of the SLC34A2 gene, additionally, heterozygous mutations were found in the parents and children of the patients (3). In order to further confirm that c.575C $>\mathrm{A}$ homozygous mutations cause the functional changes seen in PAM, in this study, we constructed a eukaryotic expression vector of the SLC34A2 gene and transfected it into human alveolar epithelial cells. The effects of the SLC34A2 and normal gene expression, respectively, on the phosphorylation of extracellular fluid by the alveolar epithelial cells was compared.

\section{Materials and methods}

Tissue and cell sources. Normal lung tissues were obtained from patients undergoing pulmonary bulla resections; PAM lung tissues were from patients undergoing CT-guided percutaneous lung biopsies that belonged to the same family. The human alveolar epithelial cells A549 were routinely preserved, recovered, passed and counted for further use in the laboratory. The study was approved by the Ethics Committee of the Second Hospital of Jilin University and informed consents were signed by the patients and/or guardians.

Construction of an SLC34A2 gene eukaryotic expression vector. Total RNA extractions from lung tissue samples were performed with a total RNA extraction kit (RNAiso Reagent, Takara Bio Inc., Dalian, China). Reverse transcription synthesis of cDNA was performed using a reverse transcription kit (D6110A, Takara Bio Inc.).

Primers and vectors were constructed according to the SLC34A2 cDNA sequence (accession no. XM_005248129) included in Genbank, two primers were designed, vector corresponding restriction endonuclease recognition sites and 
protective bases were added to the $5^{\prime}$ end of the upstream and downstream primers, respectively. The primers were synthesized and purified by the BGI, the iterated words were the endonuclease recognition sequences. The sequence for the upstream primer f was 5'-ACGGTACCTAATGGCTC CTGGCCTGAAT-3', including a KpnI endonuclease recognition site and the two protective bases. The sequence for the downstream primer $r$ was 5'-TAGCGGCCGCCTACAAG GCCGTGCATTCG-3', containing a NotI digestion recognition site (Fig. 1). The size of the target gene fragment was 2073 bp.

For PCR amplification, the reaction system included $4.0 \mu \mathrm{l}$ cDNA $+4.0 \mu \mathrm{l}$ dNTP $(100 \mu \mathrm{mol} / \mathrm{l})+1.5 \mu \mathrm{l}$ of upstream and downstream primers $(50 \mathrm{ng} / \mu \mathrm{l}$ each $)+0.5 \mu 1$ Premix Ex Taq $+5.0 \mu 1$ 10X PCR buffer (containing magnesium chloride), adding water to make the total volume reach $50 \mu \mathrm{l}$. The reaction conditions consisted of $97^{\circ} \mathrm{C}$ for $5 \mathrm{~min}$, a total of 30 cycles of $94^{\circ} \mathrm{C}$ for $50 \mathrm{sec}, 55^{\circ} \mathrm{C}$ for $55 \mathrm{sec}$, and $72^{\circ} \mathrm{C}$ for $60 \mathrm{sec}$, and then a final extension at $72^{\circ} \mathrm{C}$ for $10 \mathrm{~min}$. The amplified products were analyzed by $2 \%$ agarose gel electrophoresis to determine whether the electrophoretic bands were consistent with the design.

For extraction of gel purified products, the DNA gel recovery kit (Takara Bio Inc.) was used. Enzyme digestions were performed on the pcDNA3.1(+) plasmid (Touching Technology Co., Ltd., Shanghai, China) using restriction endonucleases KpnI and NotI (Takara Bio Inc.). Ligations were done using the DNA quick connect kit (Takara Bio Inc.). Escherichia coli DH5a competent cells (iCloning Beijing Biotech Co., Ltd., Beijing, China) were prepared and used for transformations.

Recombinant plasmids were transformed, screened and extracted using a plasmid DNA kit (DP103; Tiangen Biochemical Technology Co., Ltd., Beijing, China). The recombinant plasmid pcDNA3.1(+)-SLC34A2 was digested with KpnI and NotI and 2\% agarose gel electrophoresis was used to visualize the resulting fragments. The recombinant plasmid pcDNA3.1(+)-SLC34A2 (normal) and pcDNA3.1(+)SLC34A2 (PAM) with the target gene was conserved in glycerol, and sent to Beijing BGI for sequencing.

Cell transfection. For plasmid extractions, $20 \mu \mathrm{l}$ of bacterial liquid cultures containing pcDNA3.1(+) plasmid, pcDNA3.1(+)SLC34A2 (normal) plasmid and pcDNA3.1(+)-SLC34A2 (PAM) plasmid were inoculated into $100 \mathrm{ml} \mathrm{LB}$ medium with ampicillin, respectively, and incubated overnight for $15 \mathrm{~h}$. The plasmids were extracted according to the endotoxin free plasmid DNA Maxi kit (DP117; Tiangen Biochemical Technology Co., Ltd.). Liposome-mediated transfections of A549 cells were performed in three groups: empty vector plasmid pcDNA3.1(+) transfection (plasmid control group), normal SLC34A2 genelinked pcDNA3.1(+) recombinant plasmid transfected (PAM group), pcDNA3.1(+) plasmid recombined with PAM SLC34A2 gene (PAM group).

The transfection efficiency was detected by reverse transcription PCR (RT-PCR). RNA was extracted using the TRIzol method (Takara Bio Inc.), cDNA was synthesized with reverse transcription kit, PCR primers, amplification system and the conditions were the same as above, products were identified by electrophoresis.

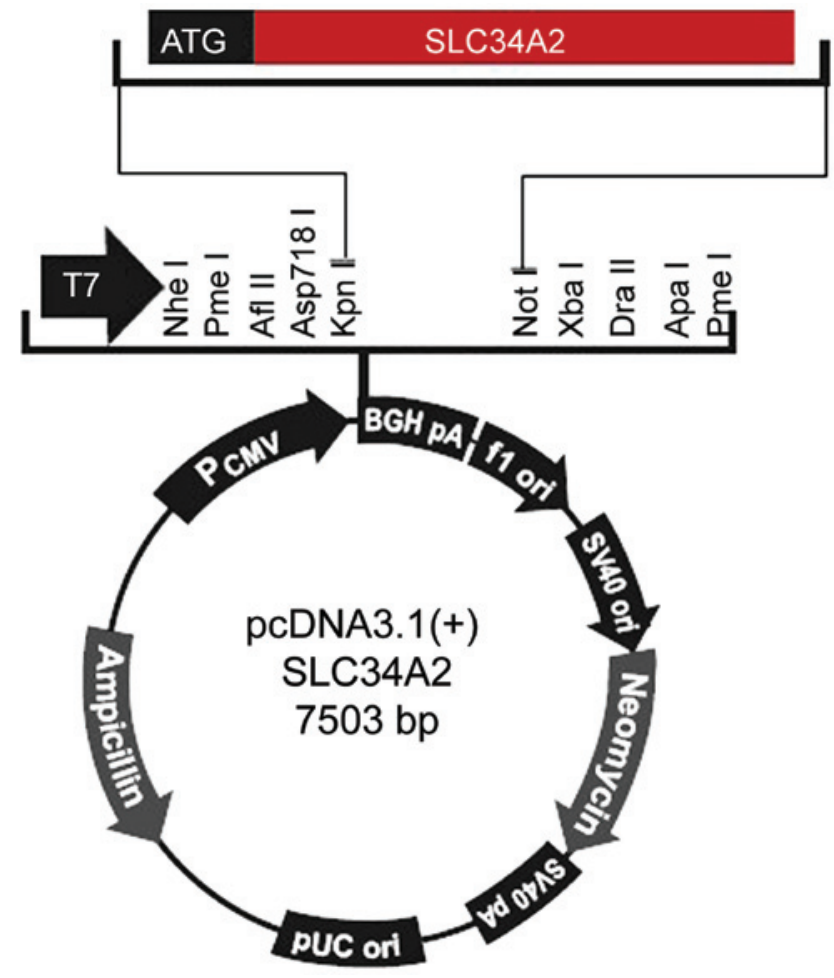

Figure 1. Recombinant plasmid SLC34A2-pcDNA3.1(+).

Detection of inorganic phosphorus concentration in culture medium. After $48 \mathrm{~h}$ of co-cultivation, the concentration of inorganic phosphorus was determined using a Hitachi 3700 automatic biochemical analyzer (Beijing no. 6 plant) using the phosphorus molybdenum blue colorimetric detection method. Measurements were repeated three times and average values were obtained for each experiment.

Statistical analysis. Statistical analysis was performed using SPSS 20.0 software (SPSS, IL, USA). The measurement data are expressed as mean \pm standard deviation. Single factor ANOVA analysis was used for comparisons among the groups, and the LSD-t method was used for multiple comparisons. A P-value of $<0.05$ was considered to indicate a statistically significant difference.

\section{Results}

RT-PCR results of SLC34A2 gene. The results showed that the amplified fragments of SLC34A2 gene in normal and PAM patients were approximately $2000 \mathrm{bp}$, in agreement with the expected size for SLC34A2 (Fig. 2).

Construction and identification of recombinant plasmids. The length of the pcDNA3.1(+) plasmid was 5428 bp, when amplifying SLC34A2 gene, 2 bp of protective bases were added, the length of the gene was $2075 \mathrm{bp}$; therefore, the length of the recombinant plasmid was calculated to be $7503 \mathrm{bp}$. After double digestion and electrophoresis, two fragments were generated. The large fragment was located at approximately $5000 \mathrm{bp}$, corresponding to the pcDNA3.1(+) plasmid fragment (5428 bp), and the small fragment was at approximately $2000 \mathrm{bp}$, which corresponded to the PCR product (2075 bp), 


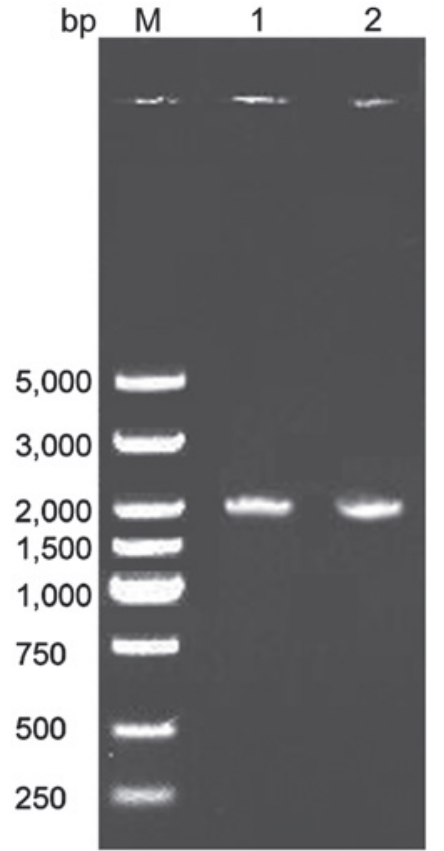

Figure 2. Electrophoresis gel after PCR amplification of SLC34A2 gene. M, Marker DL5000; lane 1, normal lung tissue; 2, PAM lung tissue.

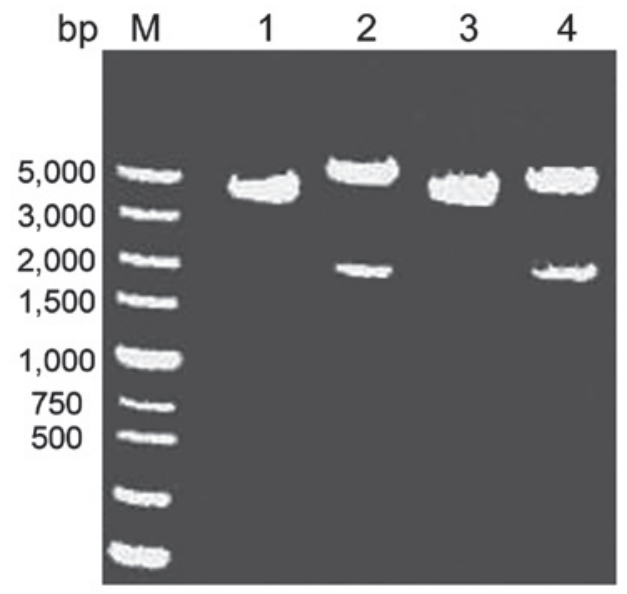

Figure 3. Electrophoresis gel after pcDNA3.1(+)-SLC34A2 digestion. M, Marker DL5000; lane 1, normal lung tissue recombinant plasmid electrophoresis results; 2 , normal lung tissue recombinant plasmid double digestion results; 3, PAM lung tissue recombinant plasmid electrophoresis results; 4, PAM lung tissue recombinant plasmid double enzyme digestion results.

indicating that the target gene fragment was successfully attached to the vector pcDNA3.1(+) (Fig. 3).

RT-PCR results of recombinant plasmid transfected cells. The results showed that the amplified fragment of SLC34A2 gene in the normal control group and PAM group was both at approximately $2000 \mathrm{bp}$, which was consistent with the size of SLC34A2. The plasmid control group did not contain the SLC34A2 gene, therefore no electrophoretic band appeared (Fig. 4).

Determination of inorganic phosphorus concentration. The concentration of inorganic phosphorus in the supernatant of the normal control group was significantly lower than that

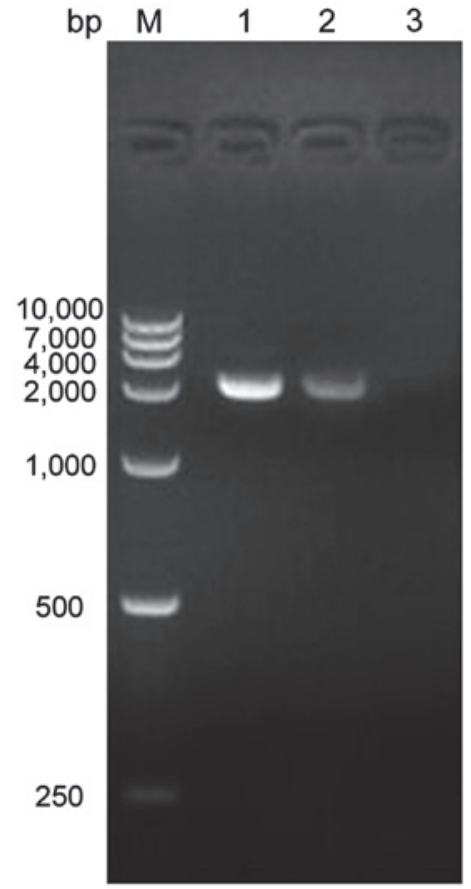

Figure 4. Electrophoresis gel after RT-PCR results from recombinant plasmid pcDNA3.1(+)-SLC34A2 transfected cells. M, marker DL10000; lane 1, normal control group; 2, PAM group; 3, plasmid control group.

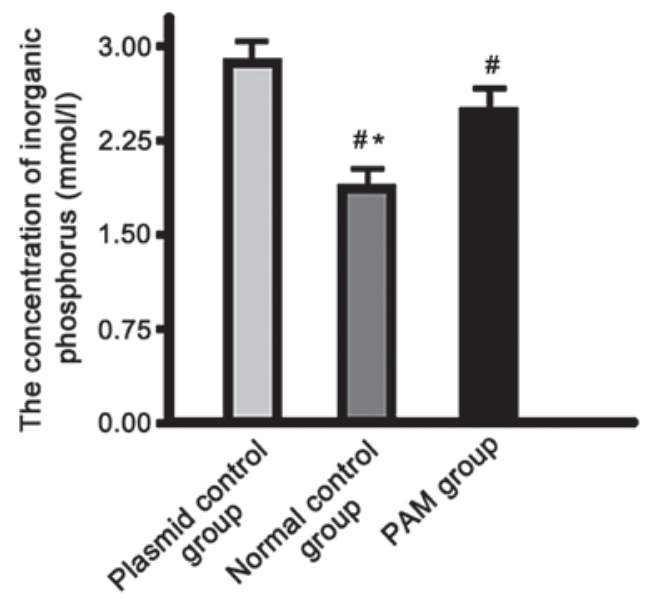

Figure 5. Determination of inorganic phosphorus concentration in culture supernatant. ${ }^{~} \mathrm{P}<0.05$ when compared to the plasmid control group. ${ }^{*} \mathrm{P}<0.05$ when compared to the PAM group.

in the plasmid control and PAM groups, and the PAM group showed significantly lower levels than the control group $(\mathrm{P}<0.01)$ (Fig. 5).

\section{Discussion}

The SLC34A2 gene has 12 exons and its total gene fragment is long (4). In order to clone the complete coding sequence of the gene, total RNA from lung tissues was extracted, reverse transcribed with Oligo (dT) and the amplified with specific primers to obtain the target gene sequence. In this way, we were able to remove the intron sequences and retain the complete coding sequence, which was needed to construct the eukaryotic expression vector in pvcDNA3.1(+). This plasmid 
contains a strong promoter of the cytomegalovirus at its $5^{\prime}$ end, the beginning of the replication site contains also the SV40 promoter, which expresses exogenous genes efficiently in mammalian cells, the expression of ampicillin and neomycin resistance genes allowed screening for the positive recombinant vector after transformation (4). The identity of our expression vectors was confirmed by restriction digestion and sequencing. The human lung adenocarcinoma cell line A549 exhibits characteristics of lung cancer malignant tumor cells and the phenotype of alveolar type II epithelial cells. The cell line is commonly used in the study of the occurrence, differentiation and structure and function of normal alveolar type II epithelial cells (5). Lipofectamine 2000 cationic liposomemediated eukaryotic cell transfections are highly efficient, and the lipofectamine technology is the most widely used transfection method employed in research these days.

The concentration of inorganic phosphorus in the supernatant of the normal control group was significantly lower than that in the plasmid control and the PAM groups, and the concentration in the PAM group was significantly lower than that in the plasmid control group. This suggests that the SLC34A2 gene mutation can reduce the phosphate transport function in PAM patients, which may be lead to the characteristic pathological changes observed in them. The most important part of inorganic phosphorus metabolism in the human body is intestinal, and intestinal inorganic phosphorus metabolism mainly relies on $\mathrm{NaPi}$-IIb. NaPi-IIb protein shows a segmental expression in the small intestine. It displays its most abundant expression in the ileal brush edge, and is little or almost undetectable in the duodenum and jejunum (6). Nicotinamide can inhibit the expression of NaPi-IIb in the brush edge of the jejunum, thereby reducing intestinal absorption of phosphorus (7); the antiviral drug foscarnet co-transports with NaPi-IIb in the small intestinal epithelial cells, competitively inhibiting phosphorus absorption, therefore reducing the level of phosphorus (8). The expression of $\mathrm{NaPi}$-IIb has been detected from the 16.5 days in the rat embryo, and the expression of $\mathrm{NaPi}$-IIb gets gradually increased during development in the lung (7). The SLC34A2 gene is expressed only in type II alveolar epithelium cells in the lungs (8), the main component of pulmonary surfactant produced by type II alveolar epithelial cells is dipalmitoyl lecithin. Phosphate is an important component of phospholipids, phosphate released by degraded phospholipids can be transported to the cells by $\mathrm{NaPi}-\mathrm{IIb}$ in the form of $\mathrm{NaH}_{2} \mathrm{PO}_{4}$ in the presence of sodium ions, that is alveolar phosphorus metabolism depends mainly on the normal function of NaPi-IIb $(9,10)$.

Our results showed that after transfection with the normal SLC34A2 gene, the resulting cells could more effectively transport the phosphate from the culture supernatant, causing the concentration of inorganic phosphorus in the culture supernatant to be significantly decreased. Furthermore, the concentrations in the supernatant were significantly lower than those in the control and PAM groups. Cells with the PAM mutated SLC34A2 gene expressed from the recombinant plasmid were still able to transport phosphate, therefore the concentration of phosphate in the medium decreased compared with that in the plasmid control group, but increased compared to that in the normal control group. Due to the SLC34A2 gene exon 6 c.575C $>$ A homozygous muta- tion in PAM patients, the original threonine is mutated into a lysine, resulting in a decreased function of the NaPi-IIb. The mutant transporter results in an impaired phosphate transport function, the phosphorus ion cannot be completely cleaned from the alveoli and phosphate is deposited in large quantities in the alveoli, where it finally combines with calcium ions to form calcium phosphate (microlithiasis) $(11,12)$. However, the transporter's function in the mutant is not completely lost, so PAM patients show no significant clinical symptoms during childhood and adolescence, when the microlithiasis is still negligible. During the asymptomatic stages, the alveolar and lobular septal structure is complete, gas exchange is not affected, and normal lung function is maintained $(13,14)$. But with the progress of the disease, calcium phosphate deposition accumulates, and microlithiasis gradually ensues. When eventually the entire alveolar cavity is filled, and the alveolar wall pressure increases, alveolar injury, inflammatory infiltration, alveolar wall thickening and interstitial fibrous tissue hyperplasia begins to appear in the alveoli, resulting in a restrictive ventilatory dysfunction and decreased diffuse function, leading to gradually deteriorating lung function $(15,16)$.

The identification of genes involved in the development of PAM has resulted in early and accurate diagnosis of sporadic cases and the diagnosis of asymptomatic family members in the PAM family. However, several traditional therapies developed according to PAM's molecular biology theory have proved to be ineffective (15). New effective treatments are sorely needed. For example, the cystic fibrosis transmembrane regulation treatment may be used for PAM patients in the future (16). Furthermore, gene therapy methods are expected to be developed as more reliable information becomes available, studies like this one contribute to the growing body of knowledge that should form the basis of such novel approaches.

\section{Acknowledgements}

The present study was supported by the Norman Bethune Program of Jilin University (2015417).

\section{References}

1. Samano MN, Waisberg DR, Canzian M, Campos SV, PêgoFernandes PM and Jatene FB: Lung transplantation for pulmonary alveolar microlithiasis: A case report. Clinics (Sao Paulo) 65: 233-236, 2010.

2. Jankovic S, Pavlov N, Ivkosic A, Erceg I, Glavina-Durdov M, Tocilj J, Dragisic-Ivulic S and Primorac D: Pulmonary alveolar microlithiasis in childhood: Clinical and radiological follow-up. Pediatr Pulmonol 34: 384-387, 2002.

3. Proesmans M, Boon M, Verbeken E, Ozcelik U, Kiper N, Van de Casseye W and De Boeck K: Pulmonary alveolar microlithiasis: A case report and review of the literature. Eur J Pediatr 171: 1069-1072, 2012.

4. Ishihara Y, Hagiwara K, Zen K, Huqun, Hosokawa Y and Natsuhara A: A case of pulmonary alveolar microlithiasis with an intragenetic deletion in SLC 34A2 detected by a genome-wide SNP study. Thorax 64: 365-367, 2009.

5. Hagiwara K, Johkoh T and Tachibana T: Molecular basis of lung disease, insight from rare lung disorders. In: Pulmonary Alveolar Microlithiasis. Panos R, Trapnell B and McCormack F (eds). Humana Press, Totowa, NJ, pp154-156, 2009.

6. Huqun Izumi S, Miyazawa H, Ishii K, Uchiyama B and Ishida T: The autozygous segments predicted by a genome-wide SNP typing revealed mutations in the type IIb sodium phosphate co-transporter (SLC34A2) causing pulmonary alveolar microlithiasis. Proc Am Thorac Soc 3: A102, 2006. 
7. Corut A, Senyigit A, Ugur SA, Altin S, Ozcelik U, Calisir H, Yildirim Z, Gocmen A and Tolun A: Mutations in SLC34A2 cause pulmonary alveolar microlithiasis and are possibly associated with testicular microlithiasis. Am J Hum Genet 79: 650-656, 2006

8. Huqun, Izumi S, Miyazawa H, Ishii K, Uchiyama B, Ishida T, Tanaka S, Tazawa R, Fukuyama S, Tanaka T, et al: Mutations in the SLC34A2 gene are associated with pulmonary alveolar microlithiasis. Am J Respir Crit Care Med 175: 263-268, 2007.

9. Izumi H, Kurai J, Kodani M, Watanabe M, Yamamoto A, Nanba E, Adachi K, Igishi T and Shimizu E: A novel SLC34A2 mutation in a patient with pulmonary alveolar microlithiasis. Hum Genome Var 4: 16047, 2017.

10. Poelma DL, Ju MR, Bakker SC, Zimmermann LJ, Lachmann BF and van Iwaarden JF: A common pathway for the uptake of surfactant lipids by alveolar cells. Am J Respir Cell Mol Biol 30: 751-758, 2004.

11. Dogan OT, Ozsahin SL, Gul E, Arslan S, Koksal B, Berk S, Ozdemir O and Akkurt I: A frame-shift mutation in the SLC34A2 gene in three patients with pulmonary alveolar microlithiasis in an inbred family. Intern Med 49: 45-49, 2010.

12. Yin X, Wang H, Wu D, Zhao G, Shao J and Dai Y: SLC34A2 Gene mutation of pulmonary alveolar microlithiasis: Report of four cases and review of literatures. Respir Med 107: 217-222, 2013.

13. Monabati A, Ghayumi MA and Kumar PV: Familial pulmonary alveolar microlithiasis diagnosed by bronchoalveolar lavage. A case report. Acta Cytol 51: 80-82, 2007.
14. Zhong YQ, Hu CP, Cai XD and Nie HP: A novel mutation of the SLC34A2 gene in a Chinese pedigree with pulmonary alveolar microlithiasis. Zhonghua Yi Xue Yi Chuan Xue Za Zhi 26: 365-368, 2009 (In Chinese).

15. Tachibana T, Hagiwara K and Johkoh T: Pulmonary alveolar microlithiasis: Review and management. Curr Opin Pulm Med 15: 486-490, 2009.

16. Ozcelik U, Yalcin E, Ariyurek M, Ersoz DD, Cinel G, Gulhan B and Kiper N: Long-term results of disodium etidronate treatment in pulmonary alveolar microlithiasis. Pediatr Pulmonol 45: 514-517, 2010.

This work is licensed under a Creative Commons Attribution-NonCommercial-NoDerivatives 4.0 International (CC BY-NC-ND 4.0) License. 